\title{
Surface Morphology of AISb on GaAs Grown by Molecular Beam Epitaxy and Real-time Growth Monitoring by in situ Ellipsometry
}

\author{
Jun Young Kim ${ }^{\mathrm{a}, \mathrm{b}}$, Ju Young Lim ${ }^{\mathrm{b}}$, Young Dong Kim ${ }^{\mathrm{a} *}$, and Jin Dong Song ${ }^{\mathrm{b} *}$ \\ ${ }^{a}$ Nano-Optical Property Laboratory and Department of Physics, Kyung Hee University, Seoul 130-701, Korea \\ ${ }^{b}$ Center for Opto-Electronic Convergence Systems, Korea Institute of Science and Technology, Seoul 136-791, Korea
}

Received November 10, 2017; revised November 29, 2017; accepted November 29, 2017

\begin{abstract}
AlSb}$ is a promising material for optical devices, particularly for high-frequency and nonlinear-optical applications. We report the effect of growth temperature on structural properties of AlSb grown on GaAs substrate. In particular we studied the surface of AlSb with the growth temperature by atomic force microscopy, and concluded that optimized growth temperature of $\mathrm{AlSb}$ is $530^{\circ} \mathrm{C}$. We also show the result of real-time monitoring of AlSb growth by in situ ellipsometry. The results of the structural study are good agreement with the previous reported ellipsometric data.
\end{abstract}

Keywords: AlSb, molecular beam epitaxy, surface morphology, real-time monitorinc, ellipsometry

\section{Introduction}

Sb-based high-electron-mobility transistors (HEMTs) have emerged as the next-generation HEMTs with advantages over conventional GaAs- or InP-based HEMTs [1]. Whereas the latter are utilized to GaAs or $\operatorname{In}(\mathrm{Ga}) \mathrm{As}$ channels and AlGaAs or InAlAs barriers, Sb-based HEMTs is applied to InAs channels and $\mathrm{Al}(\mathrm{Ga}) \mathrm{Sb}$ barriers. The advantages of this material system include the high electron mobility $\left(\sim 30,000 \mathrm{~cm}^{2} / \mathrm{Vs}\right)$ and velocity $\left(\sim 4 \times 10^{7} \mathrm{~cm} / \mathrm{s}\right)$ of bulk InAs at $300 \mathrm{~K}$ [2]. Researchers have implemented highspeed HEMT $\left(<30,000 \mathrm{~cm}^{2} / \mathrm{Vs}\right)$ with this material system and reported reduced operation voltage and power at high speed [1]. Furthermore the large conduction band offset $(1.35 \mathrm{eV})$ between InAs and AlSb [3,4] causes a strong confinement of electrons and a large $g$-factor [5]. As a result this $\mathrm{InAs} / \mathrm{Al}(\mathrm{Ga}) \mathrm{Sb}$ material system has been utilized to implement 2 dimensional electron gases (2DEGs) for spintronics applications [5-7]. Other good point of Sbbased material system is that the mobility is larger than conventional GaAs, InP and Si. Recently, researchers of Naval Research Laboratory in USA succeeded in the fabrication of Sb-based n- and p-channel heterostructure field effect transistor for high-speed and low-power application $[8,9]$.

Although theoretical calculation predicted that the mobility of InAs/Al(Ga)Sb HEMT can reach up to > $70,000 \mathrm{~cm}^{2} / \mathrm{Vs}$, the mobility of these HEMTs is limited up to less than the half of the calculation. These results from

*Corresponding author

E-mail: jdsong@kist.re.kr, ydkim@khu.ac.kr defects such as imperfection of interface [10]. The defects are attributed mainly to the formation of AlAs-like bonds between InAs and $\mathrm{Al}(\mathrm{Ga}) \mathrm{Sb}$ interface and the propagation from wafers. Also lattice mismatch between InAs and AlSb may be contributed to the defects. In the former case, a migration-enhanced epitaxy method was used between InAs and AlSb to achieve InSb-like bonds [11,12]. However there is a big huddle to solve the latter because there is no high-quality insulating substrate for InAs, $\mathrm{GaSb}$, and AlSb so-called as $0.61 \mathrm{~nm}$ lattice constant system. Although InAs and $\mathrm{GaSb}$ wafers are commercially available, those are not insulating substrates. Up to now, the only way to acquire the insulating substrate is metamorphic growth of AlSb on GaAs. The role of AlSb layer is of prime importance in overcoming the lattice mismatch $-8 \%$ between GaAs substrate and InAs/AlSb 2DEG channel-and thereby improving the transport characteristics of the HEMT structures. Although techniques such as incorporation of short period superlattices in AlSb layer [13] helped to increase the quality of the wafer, the study of the growth of $\mathrm{AlSb}$ on GaAs is critical to enhance the quality of wafer and HEMTs.

However ex-situ analysis of AlSb is very difficult because of its high reactivity to oxygen, and only few studies of AlSb have been reported. In this work we report the effects of growth temperature on structural properties of AlSb layer on GaAs. In particular, surface morphology of AlSb layer grown on GaAs with various growth temperatures is studied using x-ray diffraction (XRD) and atomic force microscopy (AFM). Also, we performed the real-time monitoring of $\mathrm{AlSb}$ growth using in situ spectroscopic ellipsometry (SE). 


\section{Experimental}

The samples were grown about $1.5 \mu \mathrm{m}$ thick on semiinsulating (0 0 1) GaAs substrate using Riber Compact 21E solid-source molecular beam epitaxy (MBE) system. We used the rotating compensator SE with the charge-coupled device (CCD) to measure the ellipsometric parameter for real time monitoring of growth process, and strain free windows to prevent distortions of the polarization states of the incoming and outgoing beams. The angle of incidence was $69.8^{\circ}$. The surface oxide of GaAs substrate was removed by heating the sample to $620^{\circ} \mathrm{C}$ under $\mathrm{As}_{2}$ flux. Then about $200 \mathrm{~nm}$ thick buffer layer of GaAs was subsequently grown at $580^{\circ} \mathrm{C}$. Changing the temperature from $580^{\circ} \mathrm{C}$ to the growth temperature $\left(T_{g}\right), \mathrm{As}_{2}$ flux was maintained with the shutter for Ga being closed and the reflection high-energy electron diffraction (RHEED) pattern remaining at $(2 \times 4)$. Reaching the preset $T_{g}$, As shutter was promptly closed with $\mathrm{Sb}$ shutter opened. It resulted in the change of RHEED pattern from $(2 \times 4)$ to $(1$ $\times 3$ ). We also performed the SE measurement and compared with the change of RHEED pattern. In the subsequent growth process of AlSb layer with the $\mathrm{Al}$ shutter opened, the RHEED pattern of 3D appeared for about $20 \mathrm{~s}$ before recovering to $(1 \times 3)$. We performed the experiment at the various $T_{g}$ from 455 to $555^{\circ} \mathrm{C}$.

The growth rate of AlSb and InAs were $0.32 \mathrm{ML} / \mathrm{s}$ and $0.23 \mathrm{ML} / \mathrm{s}$, respectively. We used the Riber's valved cracker arsenic source with the tip temperature of $900^{\circ} \mathrm{C}$ to produce $\mathrm{As}_{2}$, and the Veeco's (formerly Applied EPI) valved cracker antimony source with the tip temperature of $900^{\circ} \mathrm{C}$ to produce $\mathrm{Sb}_{2}$. Beam equivalent pressure of $\mathrm{As}_{2}$ was $\sim 6 \times 10^{-5}$ torr and flux of $\mathrm{Sb}_{2}$ was $\sim 2 \times 10^{-6}$ torr. The flux of $\mathrm{Sb}_{2}$ is following that of Ref. 14 .

\section{Results and Discussion}

Figure 1 show the real time measured SE spectra of AlSb on GaAs in the growth process. In Fig. 1(a) the change of ellipsometric parameter $\Delta$ is observed. The $\Delta$ contains the thickness information of the sample, and it changes in a periodic from 0 to $180^{\circ}$ with growth. The significant change of $\Delta$ at $\sim 0.4$ min means that the growth of AlSb has been started. Figure 1(b) shows the changes of dielectric function with time from 0.74 to $6.45 \mathrm{eV}$. These changes mean phase transition from dielectric function of $\mathrm{GaAs}$ to $\mathrm{AlSb}$ at $\sim 0.44 \mathrm{~min}$. Figure 2 show the observed RHEED patterns in the growth process. The observed RHEED pattern of GaAs is $(2 \times 4)$, and the pattern changed into (1 $\times 3$ ) with starting the growth of AlSb. This means that the RHEED pattern is in agreement with the results of SE measurement.

The surfaces of AlSb layers grown at the various $T_{g}$ were measured by AFM as shown in Fig. 3. The root mean

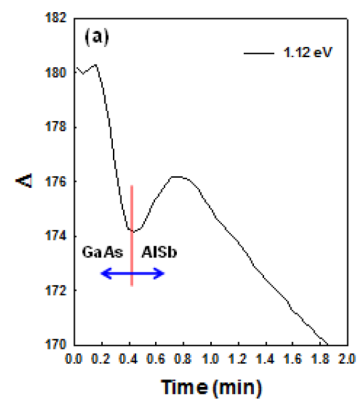

(a)

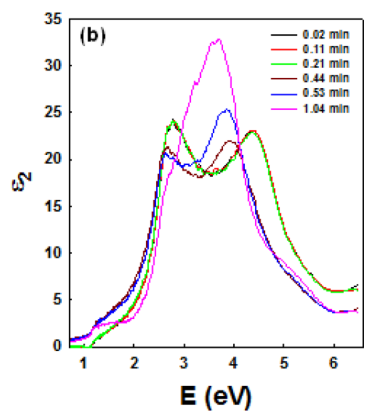

(b)
Figure 1. (a) Ellipsometric parameter $\Delta$ at $1.12 \mathrm{eV}$ during AlSb growth, (b) Pseudodielectric functions for different thicknesses of AlSb on GaAs at growth temperature.

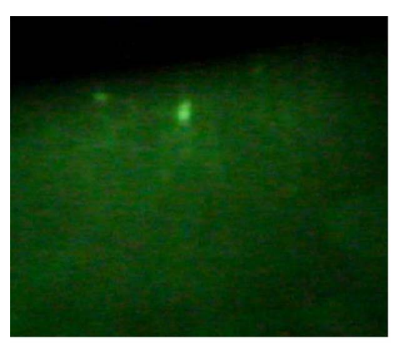

(a)

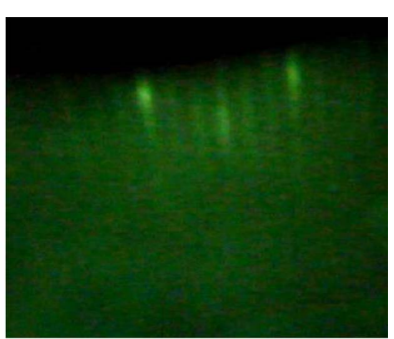

(b)
Figure 2. (a) RHEED pattern from GaAs substrate before AlSb growth, (b) RHEED pattern from AISb layer after AISb growth.

square (RMS) roughness of the surface is plotted versus the $T_{g}$ in Fig. 4 (a) and the smoothest surface with the RMS roughness of $0.619 \mathrm{~nm}$ was observed at the $T_{g}$ of $530^{\circ} \mathrm{C}$. It is a good agreement with the result of Ref. 15 and supports that the well-known optimized $T_{g}$ of AlSb layer on GaAs is $530 \sim 540^{\circ} \mathrm{C}$. It is noted that this value of the RMS roughness is comparable to the typical value of $0.4 \mathrm{~nm}$ for the surface of GaAs substrate. At the $T_{g}$ lower than $530^{\circ} \mathrm{C}$ the RMS roughness is seen to increase substantially with decreasing $T_{g}$-exhibiting a three-fold increase below $480^{\circ} \mathrm{C}$. Moreover, in Fig. 3, closer examination of the AFM images for the $T_{g}$ lower than $530^{\circ} \mathrm{C}$ appears "ripple" patterns lying parallel to the direction of $\left(\begin{array}{lll}0 & 0 & 4\end{array}\right)$. The formation of these stripe-shaped ripples may have been caused by inhomogeneous bonding between $\mathrm{Al}$ and $\mathrm{Sb}$ atoms due to shorter mean-free-path of $\mathrm{Sb}$ atoms at below $530^{\circ} \mathrm{C}$. The similar phenomenon was reported in Ref. 16 . $1.5 \mathrm{~nm}$ thick $\mathrm{GaSb}$ on InAs with In-Sb like interface grown at $350^{\circ} \mathrm{C}$-where the optimal temperature of $\mathrm{GaSb}$ on InAs is $400^{\circ} \mathrm{C}$-shows ripples on the AFM image. Although the thickness of $1.5 \mathrm{~nm}$ is too thin compared with the one of $1.5 \mu \mathrm{m}$ of AlSb, the observation of the ripple with the large RMS value $(\sim 1.2 \mathrm{~nm})$ only at lower $T_{g}$ of $350{ }^{\circ} \mathrm{C}$ and small $\mathrm{Ga} / \mathrm{Sb}$ ratio (0.81) compared to unity support that this phenomenon is attributed to the short mean-free-path of $\mathrm{Sb}$, because we can assume that the mean-free-path of $\mathrm{Ga}$ is nearly identical at both $T_{g}$ of 350 and $400^{\circ} \mathrm{C}$ and the ripple is related to localized element with shorter mean- 

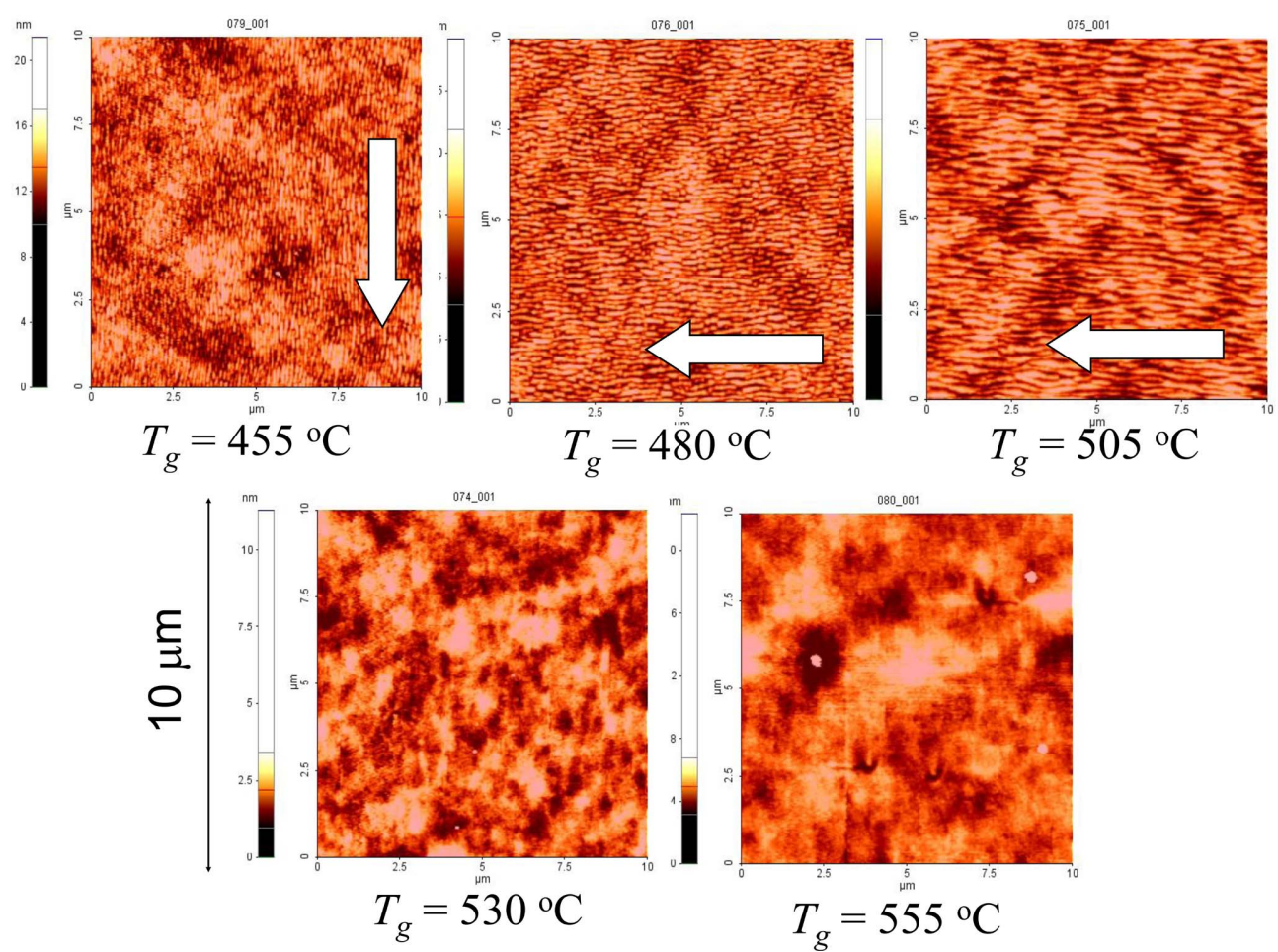

Figure 3. $10 \mu \mathrm{m} \times 10 \mu \mathrm{m}$ AFM images of $1.5 \mu \mathrm{m}$ thick AlSb layers grown on GaAs at the various growth temperatures $\left(T_{g}\right)$. No ripples were found at $T_{g} \geq 530^{\circ} \mathrm{C}$. The arrows in the figures show the direction of ripples laying parallel to (1 10$)$.

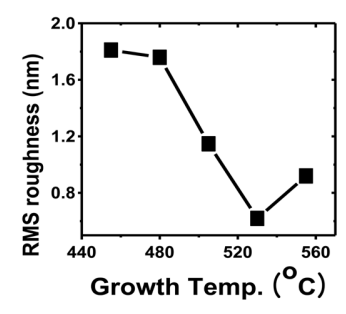

(a)

(c)

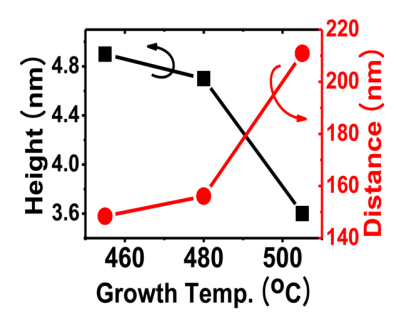

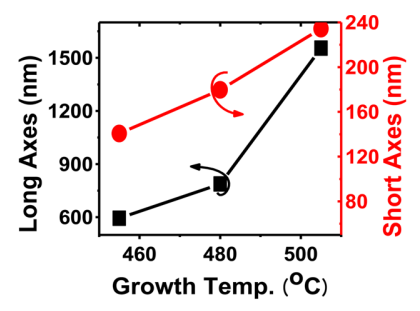

(b)

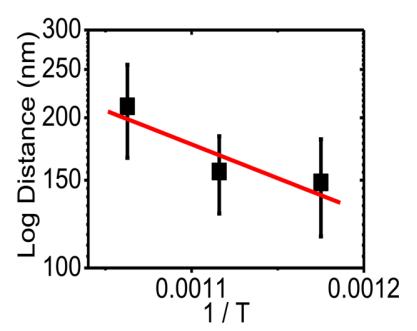

(d)
Figure 4. (a) Plot of the RMS roughness versus $T_{g}$, (b) plot of length (long axis) and width (short axis) of the observed ripples versus $T_{g}$ below $530^{\circ} \mathrm{C}$, and (c) plot of height and distance of ripples versus $T_{g}$ below $530^{\circ} \mathrm{C}$. No ripples were found at $T_{g} \geq 530^{\circ} \mathrm{C}$. (d) Arrhenius plots of distance between ripples vs. growth temperature in $\mathrm{K}$ in for $460-500^{\circ} \mathrm{C}$ grown samples.

free-path, which, in turns, shows lower $\mathrm{Ga} / \mathrm{Sb}$ ratio. It is also mentioned that $\mathrm{Ga} / \mathrm{Sb}$ ratio is nearly unity $(\sim 0.98)$ at $T_{g}=400^{\circ} \mathrm{C}$ with flat $\mathrm{GaSb} / \mathrm{InAs}$ surface at this $T_{g}[16]$.

The average lengths of long- and short-axes of the ripples were plotted versus the $T_{g}$ below $530^{\circ} \mathrm{C}$ in Fig. 4(b). The average height and the distance between ripples are also plotted in Fig. 4(c). In Fig. 4(b) and Fig. 4(c) the average size of ripples and the separation distance between them decrease with decreasing $T_{g}$ below $530^{\circ} \mathrm{C}$ while the average height increase. These result in more uneven surface with the greater RMS roughness. This phenomenon of local piling up of AlSb reflects that the materials on the surface have a lower energy to travel across the surface at the lower $T_{g}$.

On the other hand, at the $T_{g}$ higher than $530^{\circ} \mathrm{C}$, the appearance of drops and dislocations was observed in the AFM images in Fig. 3. The formation of these defects is the consequence of re-evaporation of $\mathrm{Sb}$ atoms from the surface leading to anion deficiency, i.e. V/III ratio less than unity. In addition, the RMS roughness at the $T_{g}$ higher than $530^{\circ} \mathrm{C}$ increases in Fig. 3(a).

According to the results of surface-morphological study, we conclude that the most appropriate $T_{g}$ is $\sim 530^{\circ} \mathrm{C}$ for the growth of AlSb on GaAs. Here it should be pointed out that our result is a good agreement with the result of the researchers in Naval Research Laboratory $[14,15]$ because we used the same $\mathrm{Sb}$ valved cracker and the same flux of $\mathrm{Sb}_{2}$. Therefore if the flux of $\mathrm{Sb}_{2}$ and type of $\mathrm{Sb}$ source are modified, the optimal $T_{g}$ will be changed. However the trend of surface roughness as the function of $T_{g}$ revealed in the article will be valid again. In addition it is a good agreement that the optimized $T_{g}$ is $530^{\circ} \mathrm{C}$ by previous reported SE data of AlSb [17]. From the figure 4(d), the activation energy of surface diffusion can be extracted using the following equation; $\mathrm{D}=\mathrm{D}_{\mathrm{o}} \exp \left(-\mathrm{Es} /\left(\mathrm{K}_{\mathrm{b}} \mathrm{T}\right)\right)$ where $\mathrm{D}, \mathrm{D}_{\mathrm{o}}, \mathrm{Es}, \mathrm{K}_{\mathrm{b}}$ and $\mathrm{T}$ stand for distance between ripples, constant, surface diffusion activation energy, 


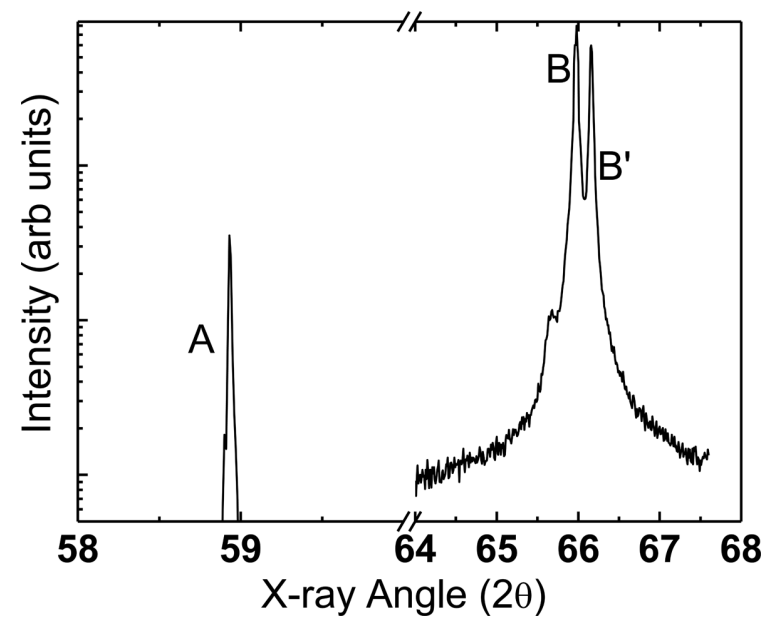

Figure 5. XRD curve of $1.5 \mu \mathrm{m}$ thick AISb layer grown on GaAs buffer layer.

substrate temperature in $\mathrm{K}$, respectively. Calculated Es is $1.356 \mathrm{eV}$ from AlSb ripples.

XRD measurement of the $\left(\begin{array}{lll}0 & 0 & 4\end{array}\right)$ reflection for typical, $1.5 \mu \mathrm{m}$ thick AlSb layer on GaAs is shown in Fig. 5. The largest double peaks (marked as B and B') near $66^{\circ}$ are from GaAs. The splitting is caused by $\mathrm{K}_{\alpha 1}$ and $\mathrm{K}_{\alpha 2}$ lines of unfiltered $\mathrm{x}$-ray source, and the deeps within the peaks are due to saturation of the detector. The single, strong peak at $58.9^{\circ}$ (marked as A) is from AlSb. Since the simulated position of $\left(\begin{array}{lll}0 & 0\end{array}\right)$ peak is $60.3^{\circ}$ for single-crystalline AlSb with $100 \%$ relaxation while the theoretical estimation of that is $55.6^{\circ}$ for fully-strained AlSb on GaAs substrate with $0 \%$ relaxation, the observed peak at $58.9^{\circ}$ in Fig. 5 corresponds to a coherent relaxation of about $71 \%$. This value is smaller than that of Ref. $14(\sim 89 \%)$, and comparable with that of Ref. 18 ( $70 \%)$. Variations of the $T_{g}$ in the range of $455 \sim 555^{\circ} \mathrm{C}$ did not have any noticeable effect on XRD spectra. In the fabrication of all 2DEGs, the $T_{g}$ of $530^{\circ} \mathrm{C}$ was therefore used for the growth of AlSb on GaAs.

Also the full width at half maximum of XRD peak for $1.5 \mu \mathrm{m}$ thick AlSb is $\sim 0.02^{\circ}$. This result is comparable with the results of Ref. 15 for $1.7 \mu$ m thick AlSb with $0.0638^{\circ}$ of peak and Ref. 19 for $1.0 \mu \mathrm{m}$ thick AlSb with $0.13^{\circ}$ of peak.

\section{Conclusions}

Antimonide-based InAs/AlSb HEMT structures were fabricated using MBE and morphological study of AlSb buffer layers and transport properties of various structures were carried out. We performed the real-time monitoring of AlSb growth by using SE measurements, and it is a good agreement with the results of RHEED pattern. This fact proves the importance and the sensitivity of SE technique for the real-time monitoring of film growth using the ellipsometry. From morphological study by AFM, it is concluded that AlSb layer on GaAs has the smoothest surface with the RMS roughness of $0.619 \mathrm{~nm}$ when the growth temperature is $530^{\circ} \mathrm{C}$. Drops and misfit dislocations are developed when the growth temperature is higher than $530^{\circ} \mathrm{C}$, and stripe-shaped pattern of ripples are observed below $530^{\circ} \mathrm{C}$. Analysis of XRD pattern measurements yield about $71 \%$ relaxation of AlSb layer grown on GaAs buffer layer. We compared this structural study of AlSb with the previous reported SE data, and concluded a good agreement that the optimized temperature of growth is $530^{\circ} \mathrm{C}$. These results will be useful database for Sb-based optoelectronic applications and high speed devices.

\section{Acknowledgements}

The authors acknowledgement the institutional program of KIST.

\section{References}

[1] B. R. Bennett, R. Magno, J. B. Boos, W. Kruppa, and M. G. Ancona, Solid-state Electronics. 49, 1875 (2005); this paper reviews $\mathrm{Sb}$-based semiconductors for electronic devices.

[2] Z. Dobrovolskis, K. Grigoras, and A. Krotkus, Appl. Phys. A: Solids Surf. 48, 245 (1989).

[3] I. Vurgaftman, J. R. Meyer, and L. R. Ram-Mohan, J. Appl. Phys. 89,5815 (2001).

[4] A. G. Milnes and A. Y. Polyakov, Mater. Sci. Eng. B 18, 237 (1993).

[5] Yu. G. Sadofyev, A. Ramamoorthy, B. Naser, J. P. Bird, S. R. Johnson, and Y-H. Zhang, Appl. Phys. Lett. 81, 1833 (2002).

[6] P. R. Hammar, and M. Johnson, Phys. Rev. Lett. 88, 066806-1 (2002).

[7] A. Zakharova, I. Lapushkin, K. Nilsson, S. T. Yen, and K. A. Chao, Phys. Rev. B 73, 125337 (2006).

[8] J. B. Boos, B. R. Bennett, N. A. Papanicolaou, M. G. Ancona, J. G. Champlain, R. Bass, and B. V. Shanabrook, Electronics Letters 43 (2007) 834.

[9] B. R. Bennett, M. G. Ancona, and J. B. Boos, Mrs Bulletin 34 (2009) 530.

[10] H. Rodilla, T. González, D. Pardo, and J. Mateos, J. Appl. Phys. 105,113705 (20009).

[11] G. Tuttle, H. Kroemer and J. H. English, J. Appl. Phys. 67, 3032 (1990).

[12] B. R. Bennett, B. V. Shanabrook, and E. R. Glaser, Appl. Phys. Lett. 65, 598 (1994).

[13] S. H. Shin, J. Y. Lim, J. D. Song, H. J. Kim, S. H. Han, and T. G. Kim, J. Korean Phys. Soc. 53, 2719 (2008).

[14] B. P. Tinkham, B. R. Bennett, R. Magno, B. V. Shanabrook, and J. B. Boos, J. Vac. Sci. Technol. B 23, 1441 (2005).

[15] B. R. Bennett, B. P. Tinkham, J. B. Boos, M. D. Lange, and R. Tsai, J. Vac. Sci. Technol. B 22, 688 (2004).

[16] A. Tahraoui, P. Tomasini, L. Lassabatère, and J. Bonnet, Appl. Surf. Sci. 162-163, 425 (2000).

[17] Y. W. Jung, T. H. Ghong, J. S. Byun, Y. D. Kim, H. J. Kim, Y. C. Chang, S. H. Shin, and J. D. Song, Appl. Phys. Lett. 94, 231913 (2009).

[18] Y. C. Lin, H. Yamaguchi, E. Y. Chang, Y. C. Hsieh, M. Ueki, Y. Hirayama, and C. Y. Chang, Appl. Phys. Lett. 90, 023509 (2007).

[19] C. Nguyen, B. Brar, C. R. Bolognesi, J. J. Pekarik, H. Kroemer, and J. H. English J. Electronic Materials 22, 255 (1993). 